\title{
Integrated analysis of ischemic stroke datasets revealed sex and age difference in anti-stroke targets
}

\author{
Wen-Xing Li ${ }^{1,2}$, Shao-Xing Dai ${ }^{2,3}$, Qian Wang ${ }^{2,3}$, Yi-Cheng Guo ${ }^{2}$, Yi Hong ${ }^{4}$, Jun-Juan Zheng ${ }^{2,3}$, Jia-Qian \\ Liu $^{2,3}$, Dahai Liu ${ }^{4}$, Gong-Hua Li ${ }^{2,3}$, Jing-Fei Huang ${ }^{\text {Corresp. } 2,3,5,6}$ \\ ${ }^{1}$ Institute of Health Sciences, Anhui University, Hefei, Anhui, China \\ 2 State Key Laboratory of Genetic Resources and Evolution, Kunming Institute of Zoology, Chinese Academy of Sciences, Kunming, Yunnan, China \\ 3 Kunming College of Life Science, University of Chinese Academy of Sciences, Kunming, Yunnan, China \\ 4 Center for Stem Cell and Translational Medicine, School of Life Sciences, Anhui University, Hefei, Anhui, China \\ 5 KIZ-SU Joint Laboratory of Animal Models and Drug Development, College of Pharmaceutical Sciences, Soochow University, Kunming, Yunnan, China \\ ${ }^{6}$ Collaborative Innovation Center for Natural Products and Biological Drugs of Yunnan, Kunming, Yunnan, China \\ Corresponding Author: Jing-Fei Huang \\ Email address: huangjf@mail.kiz.ac.cn
}

Ischemic stroke is a common neurological disorder and the burden in the world is growing. This study aims to explore the effect of sex and age difference on ischemic stroke using an integrated microarray datasets. The results showed a dramatic difference in whole gene expression profiles and influenced pathways between male and female, and also in the old and young individuals. Furthermore, compared with old male, old female patients showed more serious biological function damage. However, female showed less affected pathways than male in young subjects. Functional interaction networks showed these differential expression genes were mostly related to immune and inflammation-related functions. In addition, we found ARG1 and MMP9 were up-regulated in total and all subgroups. Importantly, ILIA, ILAB, IL6 and TNF and other anti-stroke target genes were up-regulated in males. However, these anti-stroke target genes showed low expression in females. This study found huge sex and age differences in ischemic stroke especially the opposite expression of anti-stroke target genes. Future studies are needed to uncover these pathological mechanisms, and to take appropriate pre-prevention, treatment and rehabilitation measures. 
1 Integrated analysis of ischemic stroke datasets revealed sex and age difference

2 in anti-stroke targets

3 Wen-Xing Li ${ }^{1,2, \#, ~ S h a o-X i n g ~ D a i ², 3, \#, ~ Q i a n ~ W a n g ~}{ }^{2,3, \#, ~ Y i-C h e n g ~ G u o ~}{ }^{2}$, Yi Hong ${ }^{4}$, Jun-Juan Zheng ${ }^{2,3}$, Jia-

4 Qian $\mathrm{Liu}^{2,3}$, Dahai Liü,*, Gong-Hua Li ${ }^{2,3, *}$, Jing-Fei Huang ${ }^{2,3,5,6, *}$

$5 \quad{ }^{1}$ Institute of Health Sciences, Anhui University, Hefei 230601, Anhui, China

$6{ }^{2}$ State Key Laboratory of Genetic Resources and Evolution, Kunming Institute of Zoology, Chinese

7 Academy of Sciences, Kunming 650223, Yunnan, China

$8{ }^{3}$ Kunming College of Life Science, University of Chinese Academy of Sciences, Kunming 650204, 9 Yunnan, China

$10{ }^{4}$ Center for Stem Cell and Translational Medicine, School of Life Sciences, Anhui University, Hefei 11 230601, Anhui, P. R. China

$12{ }^{5}$ KIZ-SU Joint Laboratory of Animal Models and Drug Development, College of Pharmaceutical 13 Sciences, Soochow University, Kunming 650223, Yunnan, China

$14{ }^{6}$ Collaborative Innovation Center for Natural Products and Biological Drugs of Yunnan, Kunming 15 650223, Yunnan, China

$16 \quad$ \# These authors contributed equally to this work.

$17 *$ Corresponding author:

Dahai Liu (seansean2014@126.com)

19 Gong-Hua Li (ligonghua@mail.kiz.ac.cn)

20 Jing-Fei Huang (huangjf@mail.kiz.ac.cn)

21 Tel: +86087165199200

Fax: +86087165199200 


\section{Abstract}

26 Ischemic stroke is a common neurological disorder and the burden in the world is growing. This study

27 aims to explore the effect of sex and age difference on ischemic stroke using an integrated microarray

28 datasets. The results showed a dramatic difference in whole gene expression profiles and influenced

29 pathways between male and female, and also in the old and young individuals. Furthermore, compared

30 with old male, old female patients showed more serious biological function damage. However, female

31 showed less affected pathways than male in young subjects. Functional interaction networks showed these

32 differential expression genes were mostly related to immune and inflammation-related functions. In

33 addition, we found ARG1 and MMP9 were up-regulated in total and all subgroups. Importantly, IL1A,

34 ILAB, IL6 and TNF and other anti-stroke target genes were up-regulated in males. However, these anti-

35 stroke target genes showed low expression in females. This study found huge sex and age differences in

36 ischemic stroke especially the opposite expression of anti-stroke target genes. Future studies are needed to

37 uncover these pathological mechanisms, and to take appropriate pre-prevention, treatment and rehabilitation measures. 


\section{Introduction}

Stroke is a common neurological disorder which has become the second leading cause of death worldwide (Feigin et al. 2014). It is predicted that by 2030, there could be almost 12 million stroke deaths, 70 million stroke survivors, and more than 200 million disability-adjusted life-years (DALYs) lost from stroke each year (Feigin et al. 2014). The major epidemiological risk factor for stroke is hypertension. Researchers showed that even mild hypertension is strongly associated with an increased incidence of stroke, and severe hypertension will greatly increase the burden of stroke (Kim et al. 2015). In addition to hypertension, other major risk factors such as diabetes mellitus, high levels of cholesterol and triglycerides, obesity, and smoking also contribute to stroke (Kim et al. 2015; Liu et al. 2011). Stroke burden in high-income countries is very serious, and the burden of stroke increases rapidly in low-income and middle-income countries in recent years with the rapid development of social economy (Feigin et al. 2014; Kim et al. 2015).

Ischemic stroke specifically refers to central nervous system (CNS) infarction accompanied by overt symptoms, silent CNS infarction causes no known symptoms (Sacco et al. 2013). Ischemic strokes account for the vast majority (85\%) of stroke events vs. $15 \%$ for hemorrhagic strokes (Musuka et al. 2015). It is reported that the incidence, prevalence, morbidity and mortality of ischemic stroke were influenced by sex (Barker-Collo et al. 2015; Reeves et al. 2008). Furthermore, the stroke preventive care, clinical characteristics at stroke onset, pre-hospital and in-hospital delays, diagnostic and treatment procedures, drug efficacy and response, rehabilitation and post-stroke recovery were also affected by sexual dimorphism (Reeves et al. 2008). A study of Finland and Sweden population suggested males had a higher incidence of ischemic stroke and acute coronary heart disease (CHD) than females (Hyvarinen et 
60

61

62

63

64

65

66

67

al. 2010). However, Tomita et al. showed that female is a risk factor for stroke severity and unfavorable functional outcome in patients with cardioembolic stroke (Tomita et al. 2015).

It is well known that the severity of stroke increased with the growing of age. Recently, a metaanalysis showed a periprocedural hazard ratio (HR) of 2.16 for stroke and death in patients aged 65-69 years compared with patients $<60$ years, and with higher HRs of about 4.0 for patients more than 70 years for patients assigned to carotid artery stenting (CAS) (Howard et al. 2016). And the outcomes of female/male mortality ratios for stroke were also had differences in the stratified age group (Reeves et al. 2008). A Japanese study including 33,953 patients showed women are more frequently had ischemic stroke, hypertension, dyslipidemia and other cardioembolic events with the age adjustment (Maeda et al. 2013). It is noteworthy that the age of this study population was relatively older (Female vs. Male: $75.0 \pm$ 11.7 vs. $69.3 \pm 11.4$ years). A previous review showed that premenopausal women are at a lower risk of stroke compared to men in the same age. However, the incidence of ischemic stroke increases rapidly in the postmenopausal women (Appelros et al. 2009). Therefore, the interpretation of sex difference on ischemic stroke should take into account patients' age.

However, the mechanism of this apparent sex difference in ischemic stroke risk is not fully understood. A previous study suggested that there was a huge sexually dimorphic in immune cell gene expression profiles following cardioembolic stroke, and this difference becomes maximum at 24 hours after stroke (Stamova et al. 2014). To provide an overall and clearer picture on the sex and age difference of ischemic stroke, we integrated microarray data from two platforms, explored these differences from whole gene expression profiles, metabolic pathways, functional interactions and anti-stroke targets. We also investigated the outcomes of sex difference in old and young patients. 


\section{Methods}

\section{Microarray Data Collection}

Human ischemic stroke microarray datasets were searched and downloaded from NCBI-GEO database (http://www.ncbi.nlm.nih.gov/geo) and EMBL-EBI ArrayExpress database (https://www.ebi.ac.uk/arrayexpress/) with the keywords of "Ischemic Stroke" in January 2016. The data selection criteria were: (1) all datasets were genome-wide; (2) the samples of each dataset must include ischemic stroke patients and controls; (3) the number of cases and controls in each dataset must $\geq 3$; (4) non cell line samples and (5) raw data or normalized expression matrix was available. Based on the above criteria, were finally chose two datasets for our integrate analysis (GSE16561 and GSE22255). In the dataset of GSE16561 (contributed by Barr TL), a total of 63 samples (including 39 patients and 24 controls) were tested using the Illumina HumanRef-8 v3.0 Expression BeadChip. And the GSE22255 dataset (contributed by Krug T) included 20 patients and 20 controls and each sample was hybridized to Affymetrix Human Genome U133 Plus 2.0 Array. All mRNA samples were from peripheral blood mononuclear cells (PBMCs) (Barr et al. 2010a; Krug et al. 2012). Details of the two datasets see in Supplementary Table 1. All the two datasets haven't provided information about disease history (such as hypertension) or stroke subtype. We divided the samples in this datasets into old (age $\geq 60$ ) and young (age $<60)$ group. The age distribution of ischemic stroke patients and controls saw in Supplementary Table 2. There was no difference in age between male and female both in patients and controls (Supplementary Table 2).

Data Preprocessing

R v3.2.2 was performing data preprocessing. We used Robust Multichip Average (RMA) algorithm 
102 in oligo package (Carvalho \& Irizarry 2010) to normalize the raw expression data and generate

103 normalized gene expression intensity of GSE22255. Because the GSE16561 dataset provided a RMA

104 normalized gene expression matrix (Barr et al. 2010a), therefore, we download the matrix in NCBI-GEO.

105 Gene annotation, integration and renormalization of the two datasets were carried out using custom

106 written Python code (Supplementary Code). We have removed probes that with no gene annotation or that

107 matched multiple gene symbols. Next, we calculated the average expression value of multiple probe IDs

108 that matched to an official gene symbol, and took this value to represent the expression intensity of the

109 corresponded gene symbol. The renormalization method was reported in our previous publication (Li et al.

110 2016). The distributions of RMA processed and global renormalized gene expression values in two

111 studies were showed in Supplementary Figure 1.

112 Differential Expression Analysis

113 Differential expression genes analysis was using limma package (Ritchie et al. 2015) in R v3.2.2.

114 The empirical Bayes algorithm (function "eBayes") was used to detect differentially expressed genes

115 between ischemic stroke patients and controls. Differentially expressed genes were defined as the

116 absolute value of logarithmic transformed fold-change $(\log (\mathrm{FC})) \geq \log 2(1.5)$ and a $\mathrm{P}$ value $\leq 0.05$. Up-

117 and down-regulated genes were considered as $\log (\mathrm{FC}) \geq \log 2(1.5)$ and $\log (\mathrm{FC}) \leq \log 2(1.5)$, respectively.

118 Differentially expressed genes in male, female, old and young group were calculated as male patients vs.

119 male controls, female patients vs. female controls, old patients vs. old controls and young patients vs.

120 young controls. In order to verify the sex and age differences of gene expression profiles in ischemic

121 stroke were not merely related to male vs. female or age changes in gene expression, we calculated the

122 genes that differentially expressed in males vs. females and olds vs. youngs in total group. To explain the 
123

124 125 126 127

sex and age difference in ischemic stroke, we used Venn diagram to show the number of differentially expressed genes in male vs. female, old vs. young and each cases-controls groups. And we also used the Venn diagram to show the number of up- and down-regulated genes in male and female group, and old and young group.

\section{KEGG Pathways Analysis}

We used Functional Annotation Tool in DAVID Bioinformatics Resources 6.7 (Huang da et al. 2009) to perform KEGG (Kyoto Encyclopedia of Genes and Genomes) pathway enrichment analysis and a pathway with a $\mathrm{P}$ value $\leq 0.01$ was considered to be significantly enriched. We got $5,6,3,5$ and 1 significant enriched pathways in total, male, female, old and young group, respectively. Then we take the union set of enriched pathways in male and female, and old and young group, and show the pathways and related gene expression profiles.

To explore the role of age in sex difference in ischemic stroke, we classified the datasets into four groups: old male, old female, young male and young female. Each dataset included 31 (20 patients and 11 controls), 32 (21 patients and 11 controls), 16 (7 patients and 9 controls) and 24 (11 patients and 13 controls) samples, respectively. We also performed KEGG enrichment analysis in each group and focused on the difference in the pathway level and gene expression profiles in enriched pathways between male and female in the different age group.

\section{Functional Interaction Analysis}

The GeneMANIA prediction server (http://www.genemania.org/) (Zuberi et al. 2013) was used to perform functional interaction analysis. We used the differentially expressed genes list in total, male, female, old and young group as input parameters, respectively. Since many reports were based on the 
144 total ischemic patients, we analysed the total group as a reference. The server can find other genes

145 that are related to the set of input genes and produce a functional association network based on their

146 relationships, such as pathways, co-expression, co-localization, genetic interaction, physical interaction

147 and so on. We chose the relationships of physical and genetic interactions, and pathway to produce

148 function cluster analysis.

149 Anti-stroke Targets Analysis

150 Anti-stroke target genes were searched and downloaded from Thomson Reuters Integrity Database

151 (https://integrity.thomson-pharma.com/integrity). We got a total of 531 anti-stroke target genes and then

152 mapped to our screened differentially expressed genes in total and subgroups (male, female, old and

153 young group). The total group was showed as the reference. Then we compared the difference of target

genes between male and females, and old and young patients and used "barplot" function to show the

results.

Anti-stroke Targets Validation

157

Validation of the above differentially expressed anti-stroke target genes was conducted in an

independent patient cohort. Through our rigorous screening, we finally chose GSE37587 dataset

(contributed by Barr TL) for validation. In this dataset, 34 baseline ischemic stroke mRNA samples were

from peripheral blood mononuclear cells (PBMCs), and were tested using the Illumina HumanRef-8 v3.0

Expression BeadChip (Supplementary Table 1). We downloaded the RMA normalized gene expression

to validate the differentially expressed anti-stroke target genes in total and four subgroups. 
165

166

167

168

169

170

171

172

173

174

175

176

177

178

179

180

181

182

183

184

185

\section{Results}

Overview of Differentially Expressed Genes

Venn diagram of the up- and down-regulated genes showed in Figure 1. There were 55 up- and 6 down-regulated genes in the total group. In the subgroup analysis by sex, we have detected 140 up- and 18 down-regulated genes in the male group, and 150 up- and 40 down-regulated genes in the female group, respectively (Figure 1A). Furthermore, we found 38 up- and 59 down-regulated genes in the old group, and 51 up- and 1 down-regulated genes in the young group (Figure 1B). There were only 8 genes over-expressed (ARG1, CA4, CKAP4, DUSP1, FOS, MMP9, ORM1 and RGS2) and 2 genes lowexpressed (CCR7 and ID3) both in male and female group. Additionally, in old and young group only showed 6 overlapped over-expressed genes (ARG1, CA1, FAM46C, MMP9, SDPR and THBS1). Differentially expressed genes validation showed in Supplementary Figure 2. We got 305 and 532 differentially expressed genes in male vs. female and old vs. young group. However, the numbers of overlapping genes in the two groups and other cases-controls groups only were 13 and 19. Therefore, these results suggested a huge difference in the global gene expression profiles between male and female, and also in old and young in ischemic stroke, and these differences in gene expression profiles were mainly caused by disease states.

Sex and Age Difference in Pathway View

Gene expression profiles of enrichment pathways in total group showed in Supplementary Figure 3.

The Toll-like receptor signaling pathway, NOD-like receptor signaling pathway, Cytokine-cytokine receptor interaction and Chemokine signaling pathway were significantly enriched. The union enriched pathways in male and female, and old and young patients were 8 and 6 , respectively. Figure 2 displayed

PeerJ reviewing PDF | (2016:07:12288:1:0:NEW 12 Aug 2016) 
186

187

188

189

190

191

192

193

194

195

196

197

198

199

200

201

202

203

204

205

206

the gene expression profiles of enrichment pathways in each subgroups. There were 9 up-regulated genes

in NOD-like receptor signaling pathway in male patients, whereas female seemed unaffected in this

pathway. However, female patients showed more influence in Systemic lupus erythematosus pathway

than male (Figure 2A). In Cytokine-cytokine receptor interaction pathway, male patients displayed more

over-expressed genes whereas female showed more low-expressed genes. For old patients, the

Hematopoietic cell lineage, Primary immunodeficiency, Antigen processing and presentation, Systemic

lupus erythematosus and $\mathrm{T}$ cell receptor signaling pathway were severely affected. However, only NOD-

like receptor signaling pathway was significantly affected in young patients (Figure 2B).

We further analyzed sex difference in the ischemic stroke stratified by old and young patients

(Supplementary Figure 4). The results showed that old female patients had multiple pathways seriously

down-regulated (including Hematopoietic cell lineage, NOD-like receptor signaling pathway, Cytokine-

cytokine receptor interaction, $\mathrm{T}$ cell receptor signaling pathway, Primary immunodeficiency and other pathways). However, old male patients showed the opposite results that several genes were overexpressed in the above pathways. Overall, old female showed more affected genes and pathways than old male (Supplementary Figure 4A). In the young group, we got an interesting result that male showed more up- and down-regulated genes than female in the enriched pathways (Supplementary Figure 4B). Certainly, these influenced genes and pathways were more serious in old patients than young patients both in male and female.

\section{Function Interaction Network}

Supplementary Figure 5-9 showed the function interaction network results in total, male, female, old and young group. The results showed a larger difference in the function interactions differentially 
207 208

expressed genes between male and female patients (Supplementary Figure 6-7), and also in old and young patients (Supplementary Figure 8-9). Table 1 showed top 5 enriched functions based on function interaction network in total and each subgroups. The enriched functions in total, male, female and old group were mostly related to immune and inflammation-related functions. However, in young patients showed more angiogenesis and vasculature development functions.

Mapped Anti-stroke Targets in Different Group

We mapped 5, 18, 5, 3 and 7 anti-stroke targets from differentially expressed genes in total, male, female, old and young group, respectively. These targets were shown with the yellow cycle in functional interaction network (Supplementary Figure 5-9). Figure 3 showed gene expression logFC of these targets in each group. MMP9 was over-expressed in the total and all subgroups (Figure 3A, 3B and 3C). IL1A, IL1B, IL6 and IL8 were significantly up-regulated in male patients. However, these targets showed lowexpressed in female patients. Furthermore, PDK4, HSPA1A and TLR4 were over-expressed in female patients whereas these targets were unaltered in male patients (Figure 3B). In addition, targets of PDK4, HSPA1A, JUN, IL8, SOD2, JUNB and FOSB showed a different expression status in old and young patients (Figure 3C).

\section{Validation of Anti-stroke Targets}

Validation of anti-stroke target genes in the total and four subgroups showed in Supplementary Figure 10. Similar to the above findings, IL1A, IL1B, IL6, IL8, TNF and other 10 anti-stroke target genes were significantly up-regulated in male patients. However, only PDK4 was over-expressed, other target genes had no difference in the female group. Further, JUN, IL8, SOD2, PTGS2, JUNB and FOSB were up-regulated in the old group and these targets showed no difference in the young group. 
228

229

230

231

232

233

234

235

236

237

238

239

240

241

242

243

244

245

246

247

248

\section{Discussion}

The present study showed a huge difference of gene expression profiles in male and female, and also in old and young ischemic stroke patients. These genes were mostly enriched in the immune-related pathways and cell signaling pathways. Furthermore, several anti-stroke targets showed the opposite expression between male and female.

Previous studies have made great effort on sex and age difference in ischemic stroke and got many valuable discoveries (Howard et al. 2016; Stamova et al. 2014; Tian et al. 2012). However, the sex heterogeneity of anti-stroke target genes expression still have no report. In the present study, we adopted a multi-platform integrated analysis method to conduct in deep analysis on sex and age difference in ischemic stroke especially for the anti-stroke target genes. We have also explored the interactions of sex and age on dysregulated pathways. Our study found there were more up- and downregulated genes in female than male in this study. This difference was also found in Stamova et al., which showed a more serious peripheral immune cell dysfunction in female than male at $\leq 3,5$ and $24 \mathrm{~h}$ following cardioembolic stroke (Stamova et al. 2014). Furthermore, compared with young patients, old patients showed less up-regulated genes and more down-regulated genes. In total and subgroup analysis, we found ARG1 and MMP9 were significantly higher expression in patients than controls. Indicate that these two genes were strongly associated with the pathological process of ischemic stroke. ARG1 (Arginase 1) is an essential enzyme that converts L-arginine to L-ornithine in the urea cycle. Role of ARG1 in cardiovascular disease has been studied extensively, especially in the regulation of the immune system. An increasing number of studies showed closely relations between ischemic stroke and systemic immune consequences (Asano et al. 2016). The immunosuppressive effect of ARG1 was demonstrated by 
249 El Kasmi et al., suggested ARG1 inhibition dramatically improves host immune response to tuberculosis

250 infection (El Kasmi et al. 2008). A recent study showed that stroke-related immune suppression is

251 associated with activated neutrophil and ARG1 release in middle cerebral artery occlusion (MCAO)

252 mouse (Sippel et al. 2015). Furthermore, increased expression of ARG1 in microglia following ischemic

253 stroke is linked to improved tissue remodeling and behavioral recovery (Asano et al. 2016). MMP9

254 belongs to matrix metalloproteinase family and was involved in the breakdown of extracellular matrix in

255 normal physiological processes. The association between blood brain barrier (BBB) dysfunction and

256 elevated MMP9 levels in ischemic patients was demonstrated both in a mouse model (Asahi et al. 2001)

257 and clinical studies (Barr et al. 2010b). In our study, patients with ischemic stroke showed severe immune

258 function defects. Furthermore, in anti-stroke target analysis, we found MMP9 was up-regulated in the

259

260

261

262

263

264

265

266

267

268

269

total and all subgroups. Taken together, these observations reinforce the view of severity immune and

cerebrovascular dysfunction in ischemic stroke. Additionally, mRNA samples in the present study were

all from PBMCs, thus we recommend that ARG1 and MMP9 could be used as biomarkers for the detection of ischemic stroke.

Altered gene expression is an important feature of ischemic stroke and affects proteins in numerous biological functions. This study showed different degrees of damage in NOD-like receptor signaling pathway, Hematopoietic cell lineage, Systemic lupus erythematosus and Cytokine-cytokine receptor interaction in male and female. After considering the age, more biological impairment emerged both in male and female. Especially old female presents a large number of down-regulated genes in various pathways. However, old male only showed a handful of down-regulated genes in the affected pathways (Supplementary Figure 4). The damage pathways of NOD-like receptor signaling pathway, 
270

271

272

273

274

275

276

277

278

279

280

281

282

283

284

285

286

287

288

289

290

Hematopoietic cell lineage and Cytokine-cytokine receptor interaction were also found in middle cerebral artery occlusion induced ischemic stroke mice (Quan et al. 2015). Furthermore, a recent study showed affected Ribosome, Toll-like receptor signaling pathway, MAPK signaling pathway, and Chemokine signaling pathway in the ischemic rodent brain (Liang et al. 2015). And identified three key autophagy genes (STAT3, NFKB1, and RELA) use the method of constructing the autophagy-related pathway network (Liang et al. 2015). Our results showed a slight difference of Toll-like receptor signaling pathway, MAPK signaling pathway, and Chemokine signaling pathway between male and female (Figure 2). However, when stratified by age, we observed more serious abnormal gene expression in old female than old male subjects (Supplementary Figure 4). One possible explanation of these phenomena was the role of sex hormones, for example the positive effects of estrogen on the cerebral circulation in female (Appelros et al. 2009). Some studies have indeed observed the associations between estrogen levels and the stroke risk in female and suggest that post menopausal women have a high incidence of stroke than men (Appelros et al. 2009; Reeves et al. 2008). However, randomized controlled trials among post menopausal women showed no advantages of exogenous oestrogen therapy, even increase the risk of stroke (Reeves et al. 2008). Our results found no difference in the estrogen-related pathways in females.

These results indicate that the apparent sex difference in ischemic stroke may affect by other factors.

The results of functional interaction network showed the affected proteins were mostly enriched in immune and inflammatory responses functions in total, male, female and old group. Previous studies showed several interleukins (IL1, IL6, IL8, TNF, etc.) play a crucial role in immune processes and strongly associated with ischemic stroke (Kostulas et al. 1999; Tian et al. 2012). Interleukin-1 (IL1), a highly active pro-inflammatory cytokine, acts as a main regulator of inflammation process and triggers a 
291

292

293

294

295

296

297

298

299

300

301

302

303

304

305

306

307

308

309

310

311

cascade of inflammatory mediators by activation of the IL1 receptor (Dinarello et al. 2012). There are two

related but distinct IL1 genes, IL1A and IL1B, encoding IL1 $\alpha$ and IL1 $\beta$, respectively. Experimental results show abnormal levels of IL1 $\alpha$ or IL1 $\beta$ will lead to inflammatory diseases (Dinarello et al. 2012).

IL1 is also known to involve in the neurodegeneration of acute and chronic brain disorders, including ischemic stroke, Alzheimer's disease and Parkinson's disease, although the cytokine is thought to be involved in the recovery of neurological functions (Dinarello et al. 2012; Kostulas et al. 1999). IL6 is a key early mediator of the inflammatory and overall immune response and plays an important role in the development of pathological conditions (Ferrarese et al. 1999; Ridker et al. 2000). And it's produced by many different cells including monocytes, macrophages, fibroblasts, endothelial cells, keratinocytes, mast cells, T-lymphocytes, and also by microglia and astrocytes (Ferrarese et al. 1999). An increasing number of experimental observations showed that IL6 plays a central role in the pathogenesis of several ischemic cardiovascular disorders, including ischemic stroke (Quan et al. 2015). A recent report suggested that IL6 is essential for the promoting effects of social interaction on the neurogenesis as well as long-term functional recovery after ischemic stroke (Meng et al. 2015). TNF belongs to tumor necrosis factor family and tumor necrosis factor- $\alpha(\mathrm{TNF}-\alpha)$ is the main member that has many functions including inflammation, sepsis, lipid and protein metabolism, haematopoiesis, angiogenesis and host resistance to parasites and malignancy (Beutler \& Cerami 1988; Old 1985). Previous study demonstrated that TNF- $\alpha$ activate the expression of pro-adhesive molecules on the endothelium, which causes the leukocyte accumulation, adherence, and migration from capillaries into the brain (Akira et al. 1990). Furthermore, TNF- $\alpha$ also induces the expression of IL1, IL6 and other cytokines. In the acute phase of ischemia, TNF- $\alpha$ and IL1B as inflammatory factors, cause the acceleration of inflammatory lesions, and induce cell necrosis or 
312

313

314

315

316

317

318

319

320

321

322

323

324

325

326

327

328

329

330

331

332

apoptosis (Kawai 1999).

In anti-stroke target analysis of the total group, the results showed that IL1B, IL8, JUN, MMP9 and

PTGS2 were significantly up-regulated (Figure 3A). In addition, IL1A, IL6, TNF and other anti-stroke targets were also presented an up-regulated trend. These findings were consistent with previous reports

(Kostulas et al. 1999; Ridker et al. 2000). However, after sex stratification we found that IL1A, IL1B, IL6,

IL8, TNF and other 13 anti-stroke targets were all up-regulated in male patients. Whereas most of these anti-stroke targets showed no difference or opposite expression in female patients, especially IL1A and

IL6. The change ranges of these anti-stroke targets in females apparently lower than male. Several proinflammatory cytokines, such as IL1, IL6 and TNF have been identified as promising therapeutic targets in immune and inflammatory related diseases. Extracellular acidification inhibits IL1 $\beta$ production showed improved cell environment and reduce acidosis, suggested a protective effect of chronic neurodegenerative diseases (Jin et al. 2014). IL6 has been proved as a target for the treatment of several inflammatory diseases (Metz et al. 2006). Recently, several new IL1 and IL6 inhibitors were developed and their therapeutic effects have been successfully validated (Divithotawela et al. 2016; Wiesinger et al. 2009). Furthermore, our pathway enrichment results suggested that interferon signaling may be an important factor in sex-biased mechanisms. Previous studies also provided many evidences that interferon signaling genes were closely related to stroke (Lin \& Levison 2009; Simmons et al. 2013). Thus, we extracted 23 interferon signaling genes in our datasets. Then we used GeneMANIA to perform functional interaction analysis between interferon signaling genes and differentially expressed antistroke target genes in each group. We found strong connections (including as co-expression, pathway, physical interactions, shared protein domains and co-localization) in many interferon signaling genes 
333 and anti-stroke genes. And the connection strength varies greatly in different groups (Supplementary

334 Figure 11-15).

335 Inflammation is an essential component of pathological mechanisms in charge of ischemic stroke.

336 Brazilein is an important pro-inflammatory cytokines inhibitor, it has been demonstrated a

337 neuroprotective effect though suppress TNF $\alpha$ and IL6 mRNA expressions, the treatment prospects on

338 ischemic stroke have been widely reported (Shen et al. 2007; Ye et al. 2006). Activated p38 mitogen-

339 activated protein kinase (MAPK) is closely related to cerebral ischemia disease, SB239063 is an ATP

340 competitive N-substituted imidazole-based inhibitor. Previous studies showed it has a strong inhibitory

341 effect of IL1 $\beta$, IL6, IL10, TNF and other circulating cytokines, suggested a potential therapeutic for

342 stroke patients (Bison et al. 2011; Zhang et al. 2015). Furthermore, a previous study suggested a

neuroprotective effect of benzylideneacetophenone derivatives in stroke models. These compounds act on

the JAK/STAT and MAPK pathways, inhibit mRNA expressions of IL1 $\beta$, IL6 and TNF (Jang et al. 2009).

However, these anti-stroke targets showed conversely expression between male and female in the present

study. Therefore, if researchers were not taking into account these sex differences in the investigation, the

results may conceal the real difference. And the observed increase of target genes may simply be

attributable to the differences in male. Based on our findings, we suggest that in the treatment of ischemic

stroke using these cytokine inhibitors need to be careful medication.

In conclusion, this study first demonstrated the sex and age difference in ischemic stroke use the

integrated microarray datasets. We revealed the sex difference in ischemic stroke was influenced by age.

More importantly, our results showed a conversely expression in multiple anti-stroke target genes 
354 neurological diseases, such as ischemic stroke. Therefore, in future drug development and clinical therapy

355 stage of ischemic stroke, this sex difference should be serious consideration. 


\section{References}

Akira S, Hirano T, Taga T, and Kishimoto T. 1990. Biology of multifunctional cytokines: IL 6 and related molecules (IL 1 and TNF). FASEB $J$ 4:2860-2867.

Appelros P, Stegmayr B, and Terent A. 2009. Sex differences in stroke epidemiology: a systematic review. Stroke 40:1082-1090.

Asahi M, Wang X, Mori T, Sumii T, Jung JC, Moskowitz MA, Fini ME, and Lo EH. 2001. Effects of matrix metalloproteinase-9 gene knock-out on the proteolysis of blood-brain barrier and white matter components after cerebral ischemia. J Neurosci 21:7724-7732.

Asano S, Chantler PD, and Barr TL. 2016. Gene expression profiling in stroke: relevance of blood-brain interaction. Curr Opin Pharmacol 26:80-86.

Barker-Collo S, Bennett DA, Krishnamurthi RV, Parmar P, Feigin VL, Naghavi M, Forouzanfar MH, Johnson CO, Nguyen G, Mensah GA, Vos T, Murray CJ, Roth GA, Group GBDW, and Group GBDSPE. 2015. Sex Differences in Stroke Incidence, Prevalence, Mortality and DisabilityAdjusted Life Years: Results from the Global Burden of Disease Study 2013. Neuroepidemiology 45:203-214.

Barr TL, Conley Y, Ding J, Dillman A, Warach S, Singleton A, and Matarin M. 2010a. Genomic biomarkers and cellular pathways of ischemic stroke by RNA gene expression profiling. Neurology 75:1009-1014.

Barr TL, Latour LL, Lee KY, Schaewe TJ, Luby M, Chang GS, El-Zammar Z, Alam S, Hallenbeck JM, Kidwell CS, and Warach S. 2010b. Blood-brain barrier disruption in humans is independently associated with increased matrix metalloproteinase-9. Stroke 41:e123-128.

Beutler B, and Cerami A. 1988. The Common Mediator of Shock, Cachexia, and Tumor Necrosis. Adv Immunol 42:213-231.

Bison S, Razzoli M, Arban R, Michielin F, Bertani S, and Carboni L. 2011. Effect of the p38 MAPK inhibitor SB-239063 on Lipopolysaccharide-induced psychomotor retardation and peripheral biomarker alterations in rats. Eur J Pharmacol 661:49-56.

Carvalho BS, and Irizarry RA. 2010. A framework for oligonucleotide microarray preprocessing. Bioinformatics 26:2363-2367.

Dinarello CA, Simon A, and van der Meer JW. 2012. Treating inflammation by blocking interleukin-1 in a broad spectrum of diseases. Nat Rev Drug Discov 11:633-652.

Divithotawela C, Garrett P, Westall G, Bhaskar B, Tol M, and Chambers DC. 2016. Successful treatment of cytomegalovirus associated hemophagocytic lymphohistiocytosis with the interleukin 1 inhibitor - anakinra. Respirology Case Reports 4:4-6.

El Kasmi KC, Qualls JE, Pesce JT, Smith AM, Thompson RW, Henao-Tamayo M, Basaraba RJ, Konig T, Schleicher U, Koo MS, Kaplan G, Fitzgerald KA, Tuomanen EI, Orme IM, Kanneganti TD, Bogdan C, Wynn TA, and Murray PJ. 2008. Toll-like receptor-induced arginase 1 in macrophages thwarts effective immunity against intracellular pathogens. Nat Immunol 9:13991406.

Feigin VL, Forouzanfar MH, Krishnamurthi R, Mensah GA, Connor M, Bennett DA, Moran AE, Sacco RL, Anderson L, Truelsen T, O'Donnell M, Venketasubramanian N, Barker-Collo S, Lawes CMM, Wang W, Shinohara Y, Witt E, Ezzati M, Naghavi M, and Murray C. 2014. Global and 
regional burden of stroke during 1990-2010: findings from the Global Burden of Disease Study 2010. The Lancet 383:245-255.

Ferrarese C, Mascarucci P, Zoia C, Cavarretta R, Frigo M, Begni B, Sarinella F, Frattola L, and De Simoni MG. 1999. Increased cytokine release from peripheral blood cells after acute stroke. $J$ Cereb Blood Flow Metab 19:1004-1009.

Howard G, Roubin GS, Jansen O, Hendrikse J, Halliday A, Fraedrich G, Eckstein H-H, Calvet D, Bulbulia R, Bonati LH, Becquemin J-P, Algra A, Brown MM, Ringleb PA, Brott TG, and Mas JL. 2016. Association between age and risk of stroke or death from carotid endarterectomy and carotid stenting: a meta-analysis of pooled patient data from four randomised trials. The Lancet.

Huang da W, Sherman BT, and Lempicki RA. 2009. Systematic and integrative analysis of large gene lists using DAVID bioinformatics resources. Nat Protoc 4:44-57.

Hyvarinen M, Qiao Q, Tuomilehto J, Soderberg S, Eliasson M, and Stehouwer CD. 2010. The difference between acute coronary heart disease and ischaemic stroke risk with regard to gender and age in Finnish and Swedish populations. Int J Stroke 5:152-156.

Jang S, Jung JC, Kim DH, Ryu JH, Lee Y, Jung M, and Oh S. 2009. The neuroprotective effects of benzylideneacetophenone derivatives on excitotoxicity and inflammation via phosphorylated Janus tyrosine kinase 2/phosphorylated signal transducer and activator of transcription 3 and mitogen-activated protein K pathways. $J$ Pharmacol Exp Ther 328:435-447.

Jin Y, Sato K, Tobo A, Mogi C, Tobo M, Murata N, Ishii S, Im DS, and Okajima F. 2014. Inhibition of interleukin-1 beta production by extracellular acidification through the TDAG8/cAMP pathway in mouse microglia. J Neurochem 129:683-695.

Kawai C. 1999. From myocarditis to cardiomyopathy: mechanisms of inflammation and cell death: learning from the past for the future. Circulation 99:1091-1100.

Kim AS, Cahill E, and Cheng NT. 2015. Global Stroke Belt: Geographic Variation in Stroke Burden Worldwide. Stroke 46:3564-3570.

Kostulas N, Pelidou SH, Kivisakk P, Kostulas V, and Link H. 1999. Increased IL-1beta, IL-8, and IL-17 mRNA expression in blood mononuclear cells observed in a prospective ischemic stroke study. Stroke 30:2174-2179.

Krug T, Gabriel JP, Taipa R, Fonseca BV, Domingues-Montanari S, Fernandez-Cadenas I, Manso H, Gouveia LO, Sobral J, Albergaria I, Gaspar G, Jimenez-Conde J, Rabionet R, Ferro JM, Montaner J, Vicente AM, Silva MR, Matos I, Lopes G, and Oliveira SA. 2012. TTC7B emerges as a novel risk factor for ischemic stroke through the convergence of several genome-wide approaches. J Cereb Blood Flow Metab 32:1061-1072.

Li WX, Dai SX, Liu JQ, Wang Q, Li GH, and Huang JF. 2016. Integrated Analysis of Alzheimer's Disease and Schizophrenia Dataset Revealed Different Expression Pattern in Learning and Memory. J Alzheimers Dis 51:417-425.

Liang K, Zhu L, Tan J, Shi W, He Q, and Yu B. 2015. Identification of autophagy signaling network that contributes to stroke in the ischemic rodent brain via gene expression. Neurosci Bull 31:480-490.

Lin HW, and Levison SW. 2009. Context-dependent IL-6 potentiation of interferon- gamma-induced IL12 secretion and CD40 expression in murine microglia. J Neurochem 111:808-818.

Liu L, Wang D, Wong KS, and Wang Y. 2011. Stroke and stroke care in China: huge burden, significant 
438

439

440

441

442

443

444

445

446

447

448

449

450

451

452

453

454

455

456

457

458

459

460

461

462

463

464

465

466

467

468

469

470

471

472

473

474

475

476

477

478

workload, and a national priority. Stroke 42:3651-3654.

Maeda K, Toyoda K, Minematsu K, Kobayashi S, and Japan Standard Stroke Registry Study G. 2013. Effects of sex difference on clinical features of acute ischemic stroke in Japan. J Stroke Cerebrovasc Dis 22:1070-1075.

Meng C, Zhang JC, Shi RL, Zhang SH, and Yuan SY. 2015. Inhibition of interleukin-6 abolishes the promoting effects of pair housing on post-stroke neurogenesis. Neuroscience 307:160-170.

Metz S, Wiesinger M, Vogt M, Lauks H, Schmalzing G, Heinrich PC, and Muller-Newen G. 2006. Characterization of the Interleukin (IL)-6 Inhibitor IL-6-RFP: FUSED RECEPTOR DOMAINS ACT AS HIGH AFFINITY CYTOKINE-BINDING PROTEINS. Journal of Biological Chemistry 282:1238-1248.

Musuka TD, Wilton SB, Traboulsi M, and Hill MD. 2015. Diagnosis and management of acute ischemic stroke: speed is critical. Canadian Medical Association Journal 187:887-893.

Old LJ. 1985. Tumor necrosis factor (TNF). Science 230:630-632.

Quan Z, Quan Y, Wei B, Fang D, Yu W, Jia H, Quan W, Liu Y, and Wang Q. 2015. Protein-protein interaction network and mechanism analysis in ischemic stroke. Mol Med Rep 11:29-36.

Reeves MJ, Bushnell CD, Howard G, Gargano JW, Duncan PW, Lynch G, Khatiwoda A, and Lisabeth L. 2008. Sex differences in stroke: epidemiology, clinical presentation, medical care, and outcomes. The Lancet Neurology 7:915-926.

Ridker PM, Rifai N, Stampfer MJ, and Hennekens CH. 2000. Plasma concentration of interleukin-6 and the risk of future myocardial infarction among apparently healthy men. Circulation 101:17671772.

Ritchie ME, Phipson B, Wu D, Hu Y, Law CW, Shi W, and Smyth GK. 2015. limma powers differential expression analyses for RNA-sequencing and microarray studies. Nucleic Acids Res 43:e47.

Sacco RL, Kasner SE, Broderick JP, Caplan LR, Connors JJ, Culebras A, Elkind MS, George MG, Hamdan AD, Higashida RT, Hoh BL, Janis LS, Kase CS, Kleindorfer DO, Lee JM, Moseley ME, Peterson ED, Turan TN, Valderrama AL, Vinters HV, American Heart Association Stroke Council CoCS, Anesthesia, Council on Cardiovascular R, Intervention, Council on C, Stroke N, Council on E, Prevention, Council on Peripheral Vascular D, Council on Nutrition PA, and Metabolism. 2013. An updated definition of stroke for the 21 st century: a statement for healthcare professionals from the American Heart Association/American Stroke Association. Stroke 44:2064-2089.

Shen J, Zhang H, Lin H, Su H, Xing D, and Du L. 2007. Brazilein protects the brain against focal cerebral ischemia reperfusion injury correlating to inflammatory response suppression. Eur J Pharmacol 558:88-95.

Simmons S, Lee RV, Moller T, and Weinstein JR. 2013. Thrombin induces release of proinflammatory chemokines interleukin-8 and interferon-gamma-induced protein-10 from cultured human fetal astrocytes. Neuroreport 24:36-40.

Sippel TR, Shimizu T, Strnad F, Traystman RJ, Herson PS, and Waziri A. 2015. Arginase I release from activated neutrophils induces peripheral immunosuppression in a murine model of stroke. $J$ Cereb Blood Flow Metab 35:1657-1663.

Stamova B, Jickling GC, Ander BP, Zhan X, Liu D, Turner R, Ho C, Khoury JC, Bushnell C, Pancioli A, 
Jauch EC, Broderick JP, and Sharp FR. 2014. Gene expression in peripheral immune cells following cardioembolic stroke is sexually dimorphic. PLoS One 9:e102550.

Tian Y, Stamova B, Jickling GC, Liu D, Ander BP, Bushnell C, Zhan X, Davis RR, Verro P, Pevec WC, Hedayati N, Dawson DL, Khoury J, Jauch EC, Pancioli A, Broderick JP, and Sharp FR. 2012. Effects of gender on gene expression in the blood of ischemic stroke patients. $J$ Cereb Blood Flow Metab 32:780-791.

Tomita H, Hagii J, Metoki N, Saito S, Shiroto H, Hitomi H, Kamada T, Seino S, Takahashi K, Baba Y, Sasaki S, Uchizawa T, Iwata M, Matsumoto S, Shoji Y, Tanno T, Osanai T, Yasujima M, and Okumura K. 2015. Impact of Sex Difference on Severity and Functional Outcome in Patients with Cardioembolic Stroke. J Stroke Cerebrovasc Dis 24:2613-2618.

Wiesinger MY, Haan S, Wuller S, Kauffmann ME, Recker T, Kuster A, Heinrich PC, and Muller-Newen G. 2009. Development of an IL-6 inhibitor based on the functional analysis of murine IL6Ralpha(1). Chem Biol 16:783-794.

Ye M, Xie WD, Lei F, Meng Z, Zhao YN, Su H, and Du LJ. 2006. Brazilein, an important immunosuppressive component from Caesalpinia sappan L. Int Immunopharmacol 6:426-432.

Zhang XM, Zhang L, Wang G, Niu W, He Z, Ding L, and Jia J. 2015. Suppression of mitochondrial fission in experimental cerebral ischemia: The potential neuroprotective target of p38 MAPK inhibition. Neurochem Int 90:1-8.

Zuberi K, Franz M, Rodriguez H, Montojo J, Lopes CT, Bader GD, and Morris Q. 2013. GeneMANIA prediction server 2013 update. Nucleic Acids Res 41:W115-122. 
499 Table 1. Top five enriched functions based on functional interaction network in each group.

\begin{tabular}{|c|c|c|c|}
\hline Functions & FDR & $\begin{array}{l}\text { Genes in } \\
\text { network }\end{array}$ & $\begin{array}{c}\text { Genes in } \\
\text { genome }\end{array}$ \\
\hline \multicolumn{4}{|l|}{ Total } \\
\hline response to bacterium & $1.47 \mathrm{E}-10$ & 14 & 166 \\
\hline defense response to bacterium & $6.97 \mathrm{E}-09$ & 10 & 76 \\
\hline cell chemotaxis & $1.52 \mathrm{E}-08$ & 12 & 157 \\
\hline positive regulation of defense response & $5.45 \mathrm{E}-08$ & 13 & 228 \\
\hline defense response to other organism & 7.71E-08 & 12 & 188 \\
\hline \multicolumn{4}{|l|}{ Male } \\
\hline inflammatory response & $2.43 \mathrm{E}-12$ & 24 & 282 \\
\hline cell chemotaxis & $1.53 \mathrm{E}-07$ & 15 & 157 \\
\hline negative regulation of multicellular organismal process & $1.60 \mathrm{E}-07$ & 17 & 229 \\
\hline positive regulation of defense response & $1.60 \mathrm{E}-07$ & 17 & 228 \\
\hline leukocyte migration & $1.60 \mathrm{E}-07$ & 17 & 230 \\
\hline \multicolumn{4}{|l|}{ Female } \\
\hline defense response to other organism & $1.57 \mathrm{E}-13$ & 23 & 188 \\
\hline response to bacterium & $2.19 \mathrm{E}-10$ & 19 & 166 \\
\hline defense response to bacterium & $1.10 \mathrm{E}-08$ & 13 & 76 \\
\hline negative regulation of viral genome replication & $3.25 \mathrm{E}-08$ & 10 & 38 \\
\hline secretory granule & $3.86 \mathrm{E}-08$ & 16 & 152 \\
\hline \multicolumn{4}{|l|}{ Old } \\
\hline defense response to bacterium & $5.43 \mathrm{E}-07$ & 10 & 76 \\
\hline defense response to other organism & $1.02 \mathrm{E}-06$ & 13 & 188 \\
\hline secretory granule & $1.24 \mathrm{E}-05$ & 11 & 152 \\
\hline response to fungus & $1.55 \mathrm{E}-05$ & 5 & 11 \\
\hline response to bacterium & $1.88 \mathrm{E}-05$ & 11 & 166 \\
\hline \multicolumn{4}{|l|}{ Young } \\
\hline angiogenesis & $2.57 \mathrm{E}-04$ & 10 & 237 \\
\hline regulation of vasculature development & 4.42E-04 & 8 & 146 \\
\hline inflammatory response & 4.42E-04 & 10 & 282 \\
\hline regulation of angiogenesis & $2.28 \mathrm{E}-03$ & 7 & 131 \\
\hline positive regulation of defense response & 4.58E-03 & 8 & 228 \\
\hline
\end{tabular}




\section{Up-regulation Genes}
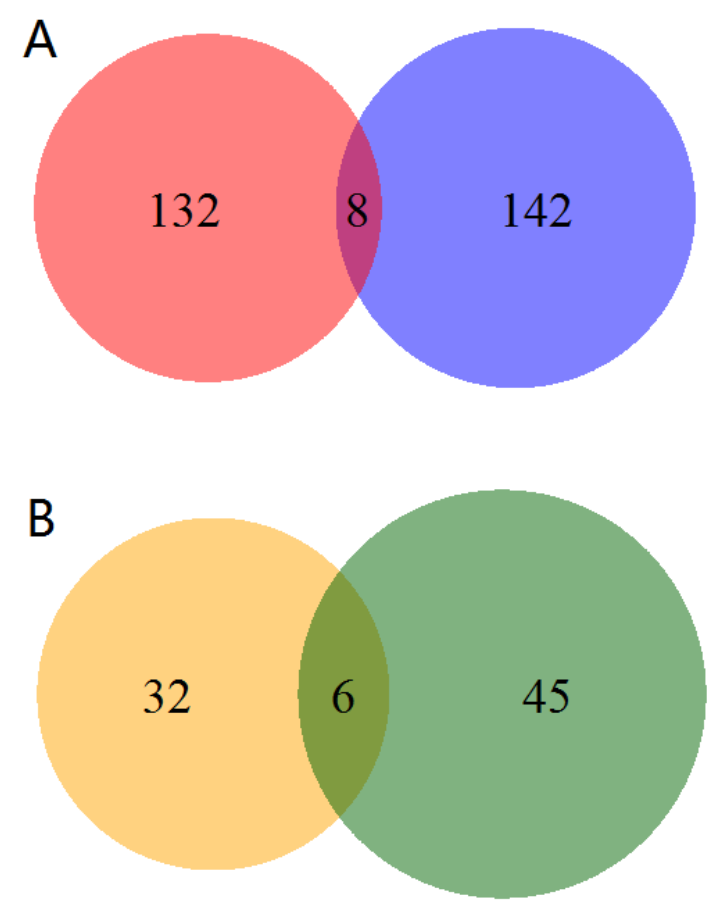

Male

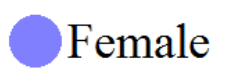

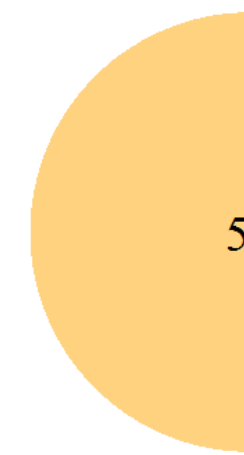

\section{Down-regulation Genes}

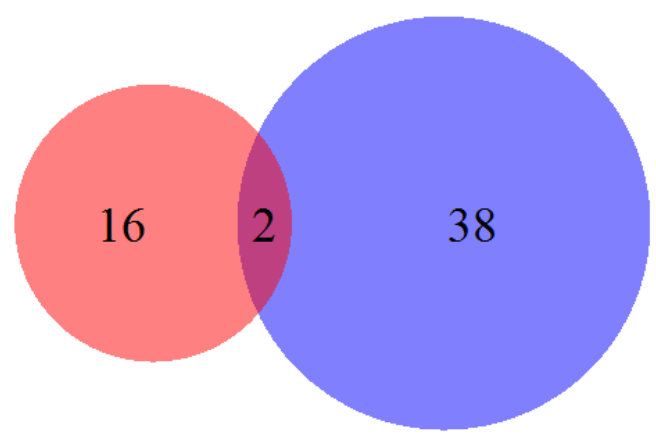

59

1

501

502 Figure 1. Venn diagram of the differentially expressed genes grouped by sex and age. Panel A showed the up- and down-regulated genes in male and female group (red and blue cycle). Panel B showed the upand down-regulated genes in old and young group (yellow and green cycle). The junction of the circle showed the overlapped genes in the two groups. 

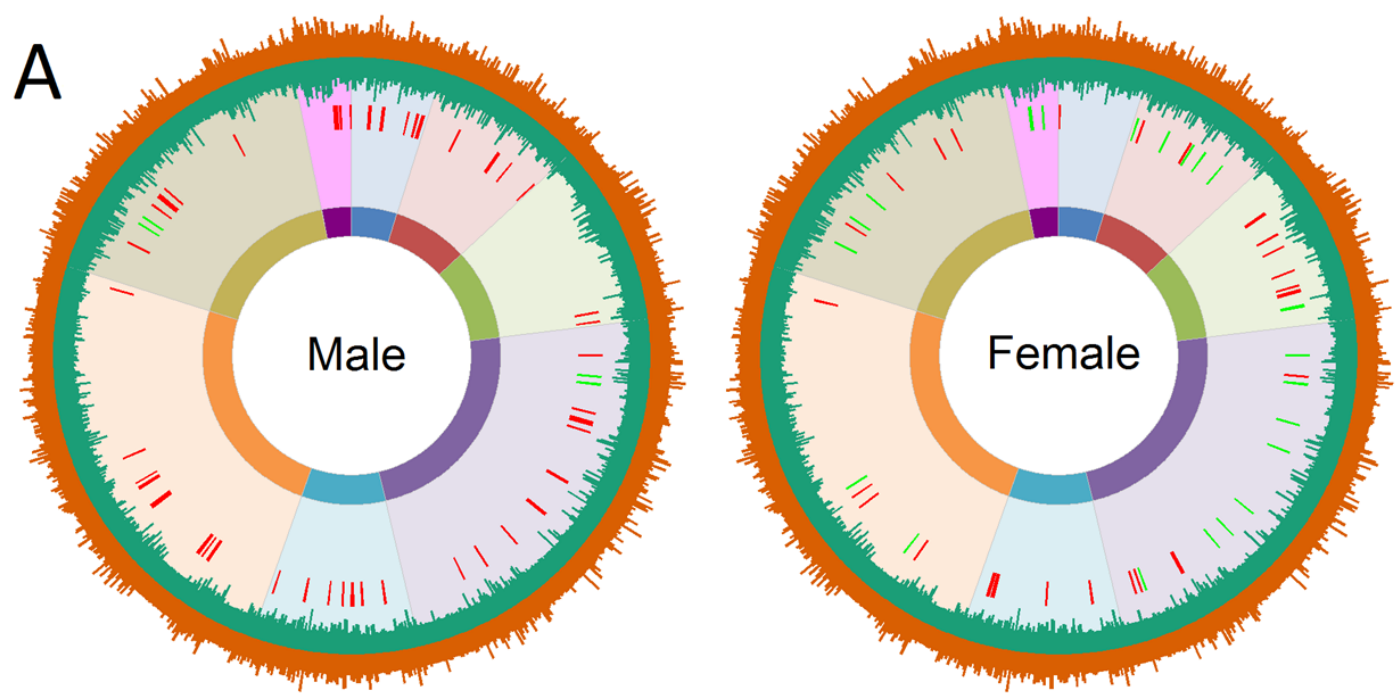

NOD-like receptor signaling pathway

- Hematopoietic cell lineage

- Ischemic Stroke

- Systemic lupus erythematosus

- Cytokine-cytokine receptor interaction

- Control

- Toll-like receptor signaling pathway

MAPK signaling pathway

Chemokine signaling pathway

- Graft-versus-host disease
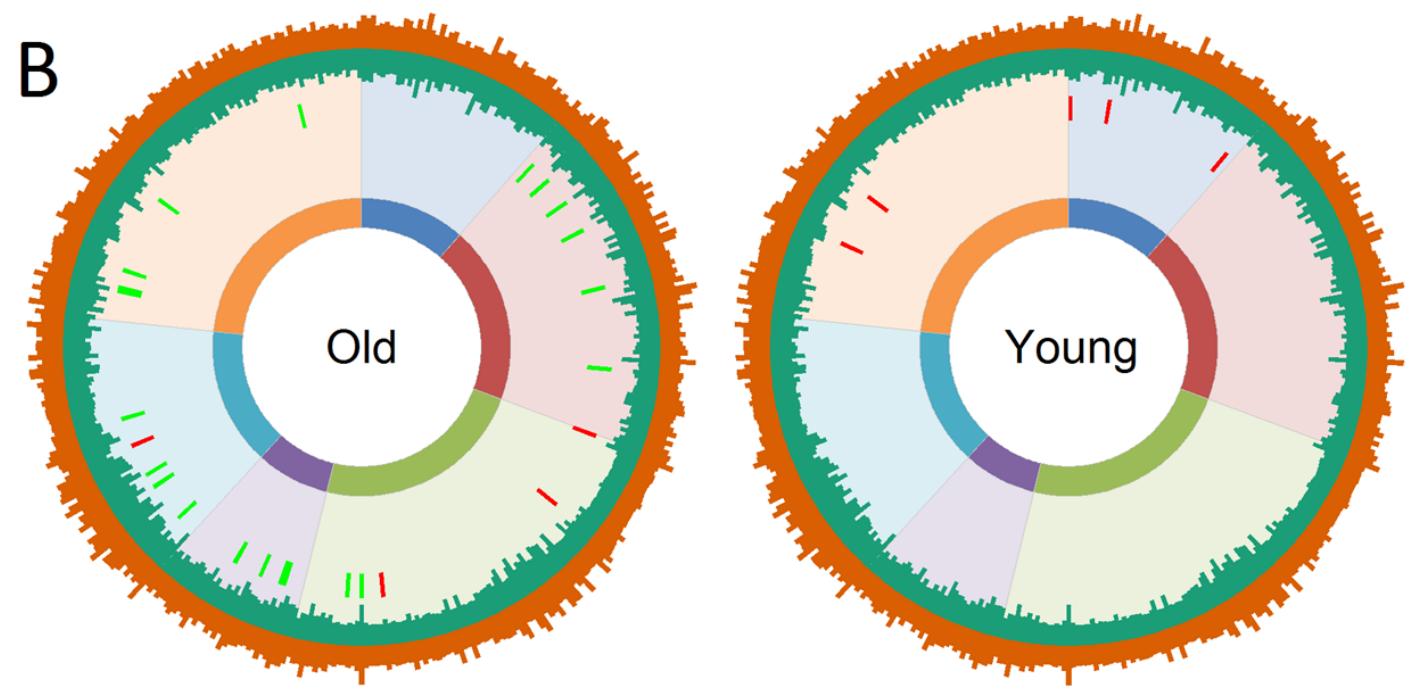

NOD-like receptor signaling pathway

- Hematopoietic cell lineage

- Ischemic Stroke

- Systemic lupus erythematosus

- Primary immunodeficiency

- Control

- Up-regulation Gene

- Antigen processing and presentation

- Down-regulation Gene

T cell receptor signaling pathway

Figure 2. Gene expression profiles of enrichment pathways. Panel A showed the gene expression profiles

in male and female group. Panel $\mathbf{B}$ showed the gene expression profiles in old and young group. Pathways 
509 are represented by different colors. The length of the first layer lines outside the circle represents the 510 expression value in ischemic stroke patients and the length of the second layer lines within the circle 511 represents the expression value in controls. The up- and down-regulated genes marked as red and green 512 lines in the third layer. 

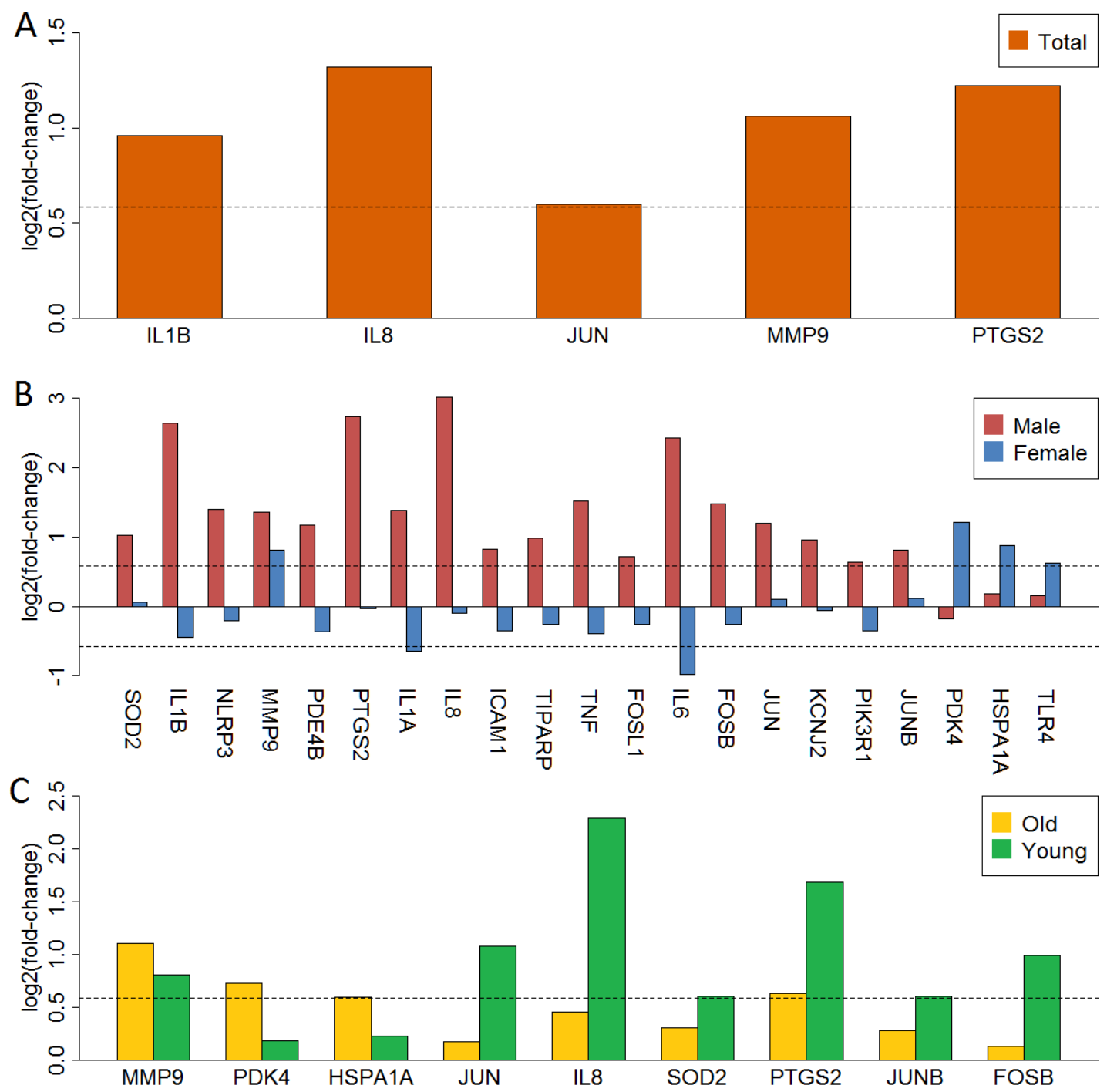

514 Figure 3. LogFC barplot of mapped anti-stroke target genes. Panel A showed the $\operatorname{logFC}$ in total group.

515 Panel $\mathbf{B}$ showed the $\log F C$ in male and female group. Panel $\mathbf{C}$ showed the $\log F C$ in old and young group.

516 The horizontal dashed lines represent the $\operatorname{logFC}$ cutoff of the up- and down-regulated genes. 